\title{
The Synthesis of Amphiphilic Luminescent Graphene Quantum Dot and Its Application in Miniemulsion Polymerization
}

\author{
Minxiang Zeng, ${ }_{1}^{1}$ Xuezhen Wang, ${ }^{1,2}$ Yi-Hsien Yu, ${ }^{3}$ Lecheng Zhang, ${ }^{1,2}$ Wakaas Shafi, \\ Xiayun Huang, ${ }^{1}$ and Zhengdong Cheng ${ }^{1,2,3}$ \\ ${ }^{1}$ Artie McFerrin Department of Chemical Engineering, Texas A\&M University, College Station, TX 77843-3122, USA \\ ${ }^{2}$ Mary Kay O’Connor Process Safety Center, Artie McFerrin Department of Chemical Engineering, Texas A\&M University, \\ College Station, TX 77843-3122, USA \\ ${ }^{3}$ Department of Material Science and Engineering, Texas A\&M University, College Station, TX 77843-3003, USA
}

Correspondence should be addressed to Zhengdong Cheng; zcheng@tamu.edu

Received 5 March 2016; Accepted 17 April 2016

Academic Editor: Paulo Cesar Morais

Copyright (C) 2016 Minxiang Zeng et al. This is an open access article distributed under the Creative Commons Attribution License, which permits unrestricted use, distribution, and reproduction in any medium, provided the original work is properly cited.

\begin{abstract}
Although emulsion applications of microscale graphene sheets have attracted much attention recently, nanoscale graphene platelets, namely, graphene quantum dots (GQDs), have been rarely explored in interface science. In this work, we study the interfacial behaviors and emulsion phase diagrams of hydrophobic-functionalized graphene quantum dots $\left(\mathrm{C}_{18}\right.$-GQDs). Distinctive from pristine graphene quantum dots ( $\mathrm{p}$-GQDs), $\mathrm{C}_{18}$-GQDs show several interesting surface-active properties including high emulsification efficiency in stabilizing dodecane-in-water emulsions. We then utilize the $\mathrm{C}_{18}$-GQDs as surfactants in miniemulsion polymerization of styrene, achieving uniform and relatively small polystyrene nanospheres. The high emulsification efficiency, low production cost, uniform morphology, intriguing photoluminescence, and extraordinary stability render $\mathrm{C}_{18}$-GQDs an attractive alternative in surfactant applications.
\end{abstract}

\section{Introduction}

In Pickering emulsions, the solid particles play a key role at the interface of two immiscible phases to prevent the coalescence by generating a mechanically robust monolayer [1]. This field received widespread scientific interests for developing stable Pickering emulsions/foam [2-4], double emulsions [5-8], liquid marbles, and superhydrophobic barriers $[9,10]$. As nanomanufacturing processes are getting mature and well-controlled, more and more nanomaterials are adopted as the stabilizers for Pickering emulsions, such as functionalized silica, titania nanoparticles, zirconium phosphate, protein microgel, clay, and polymer particles [11-21]. However, most procedures are still relatively complicated and require costly reagents for synthesizing the amphiphilic nanoparticles with desired properties, resulting in limited applications.

Carbon-based nanoparticles have attracted much attention in the past decades owing to their low cytotoxicity, chemical inertness, cost-effectiveness, and biocompatibility [2228]. Because it is also difficult to obtain the desired surface properties, there were only few works reporting graphenebased materials as surfactants [11, 29]. Recently, Yin et al. demonstrated that graphene oxide (GO) nanosheets were utilized in Pickering emulsion polymerization to form GO coated polystyrene (PS) microspheres with a narrow range of size distribution [30]. Although pristine GO nanosheets could not stabilize the styrene droplets in aqueous phase due to high hydrophilicity of GO, the hydrophobic modification by adsorbing PS oligomers on GO surface led to the nascent nuclei formation and continuous Pickering emulsion polymerization successfully. In 2014, Kim et al. reported a top-down method for synthesizing graphene quantum dots (GQDs) as surfactants [11]. Pristine GQDs (p-GQDs) were first studied and showed poor emulsification performance. By thermally reducing the oxygen-containing groups on $\mathrm{p}$ GQDs, the hydrophobicity was improved and further enabled the reduced GQDs (r-GQDs) to serve as the interface stabilizer in toluene-in-water emulsions. However, due to the limited hydrophobicity introduced by thermal reduction, the 

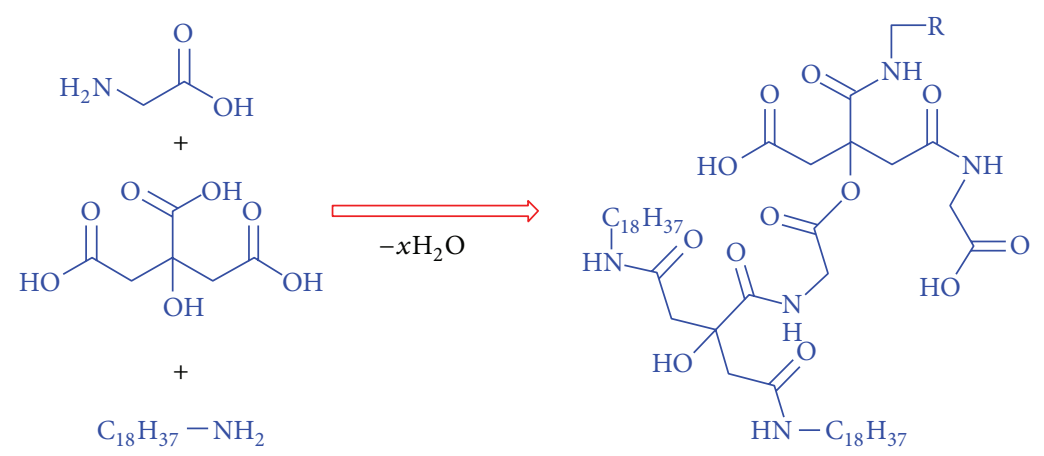

Polymer-like intermediate

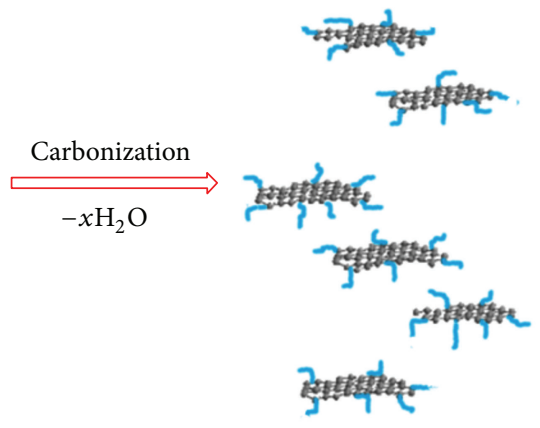

Carbogenic $\mathrm{C}_{18}$-GQDs

FIgURE 1: The schematic illustration of the formation of $\mathrm{C}_{18}$-GQDs.

average size of Pickering emulsions stabilized by r-GQDs was relatively large, and the size distribution of PS colloidal particles was much broader than that of PS colloidal particles stabilized by organic/polymer surfactant [31]. Therefore, it is desirable to develop a highly efficient particle surfactant by controlling the wettability of the amphiphilic GQDs.

An alternative method to improve hydrophobicity of nanoparticles can be achieved by introducing hydrophobic functional groups, such as alkane chain. In this study, we developed a facile bottom-up approach to synthesize octadecyl grafted luminescent graphene quantum dots $\left(\mathrm{C}_{18}\right.$-GQDs) surfactant with controllable wettability. The oleophilicity and hydrophilicity of $\mathrm{C}_{18}$-GQDs were balanced by controlling the chemical condensation of hydrophobic alkane chain. As a novel class of efficient particle stabilizers for Pickering emulsions, $\mathrm{C}_{18}$-GQDs demonstrated the ability to stabilize dodecane-in-water emulsions and were employed to produce PS/GQDs microspheres via miniemulsion polymerization of styrene. The pristine graphene quantum dots ( $\mathrm{p}-\mathrm{GQDs}$ ) were also synthesized without octadecyl group grafting for comparison.

\section{Materials and Methods}

2.1. Materials. All chemicals and solvents were used as received without further purification unless otherwise stated. Citric acid monohydrate (Sigma-Aldrich, USA), azobisisobutyronitrile (AIBN, Sigma-Aldrich, USA), glycine (Fluka, USA), octadecylamine (Acros, USA), styrene (Acros, USA), and dodecane (Acros, USA) were used.

2.2. Synthesis of $C_{18}-G Q D s$. Figure 1 shows the schematic illustration of $\mathrm{C}_{18}$-GQDs synthesis. First, $1.0 \mathrm{~g}$ of citric acid monohydrate $(4.8 \mathrm{mmol})$ was dissolved in $25 \mathrm{~mL}$ of ethanol, followed by adding an ethanol solution containing $0.75 \mathrm{~g}$ of octadecylamine $(2.8 \mathrm{mmol})$. The mixture was stirred for 1 hour, and the formed precipitate was filtered and washed with ethanol several times. After drying in an oven at $65^{\circ} \mathrm{C}$ for 24 hours, the white solid product was mixed with $0.3 \mathrm{~g}$ of glycine $(4.0 \mathrm{mmol})$ and then transferred into a $20 \mathrm{~mL}$ glass vial and calcinated in air at $200^{\circ} \mathrm{C}$ for $3 \mathrm{~h}$. The dark solid residue was further purified by column chromatography to remove unreacted starting materials for obtaining $\mathrm{C}_{18}$-GQDs. For comparison, hydrophilic p-GQDs were prepared by a previous work [23]. Specifically, the citric acid was heated in a $20 \mathrm{~mL}$ glass vial in air at $200^{\circ} \mathrm{C}$ without the addition of octadecylamine for comparison.

2.3. Contact Angle Measurement. Homogeneous $\mathrm{C}_{18}$-GQDs films were prepared by coating a few drops of $\mathrm{C}_{18}$-GQDs dispersion $(0.18 \mathrm{wt} \%)$ onto precleaned glass plates, followed by drying at $70^{\circ} \mathrm{C}$ for $4 \mathrm{~h}$. After cooling down to room temperature, one drop of deionized water was placed onto the surface and allowed to equilibrate for $20 \mathrm{~s}$ before making any measurement. Homogeneous p-GQDs films were prepared by the same procedure. A digital camera was used to record the images and their contact angles were calculated by PolyPro software package.

2.4. Preparation of $C_{18}$-GQDs Pickering Emulsions. To prepare aqueous dispersion, the purified graphene quantum dots were dissolved in $1 \mathrm{~mL}$ acetone followed by $20 \mathrm{~mL}$ deionized water. Then, the acetone was removed at $65^{\circ} \mathrm{C}$ under a reduced pressure. Finally, the $\mathrm{C}_{18}$-GQDs dispersion was centrifuged for 20 minutes at $4000 \mathrm{rpm}$ to remove possible aggregates. Part of the final dispersion was taken out to determine concentration by drying at $75^{\circ} \mathrm{C}$ in an oven for 2 days. For Pickering emulsion formation, $\mathrm{C}_{18}-\mathrm{GQDs}$ and $\mathrm{p}$ GQDs dispersions with the designed weight percentages were brought into a glass vial, and then dodecane was slowly added to the dispersion under sonication for 2 minutes.

2.5. Preparation of $C_{18}-G Q D s / P S$ Composites. First, prepare $0.9 \mathrm{~mL} \mathrm{C} \mathrm{C}_{18}$-GQDs aqueous solution $(0.18 \mathrm{wt} \%)$ in a $5 \mathrm{~mL}$ glass vial, and $0.1 \mathrm{~mL}$ styrene and $2 \mathrm{mg}$ azobisisobutyronitrile (AIBN) were then introduced. This mixture was manually shaken for 30 seconds. Finally, the entire suspension of $\mathrm{C}_{18^{-}}$ GQDs/PS precursor was transferred into a $70^{\circ} \mathrm{C}$ oven for 4 hours, allowing for the full polymerization of styrene. The products were washed with warm DI water $\left(50^{\circ} \mathrm{C}\right)$ twice and then dried using a freeze drier.

2.6. Characterization. The morphologies of $\mathrm{C}_{18}-\mathrm{GQDs}$ and $\mathrm{C}_{18}$-GQDs/PS composites were characterized by transmission electron microscopy (TEM, JEM-2010, JEOL) operated at an accelerating voltage of $200 \mathrm{kV}$ and field emission 


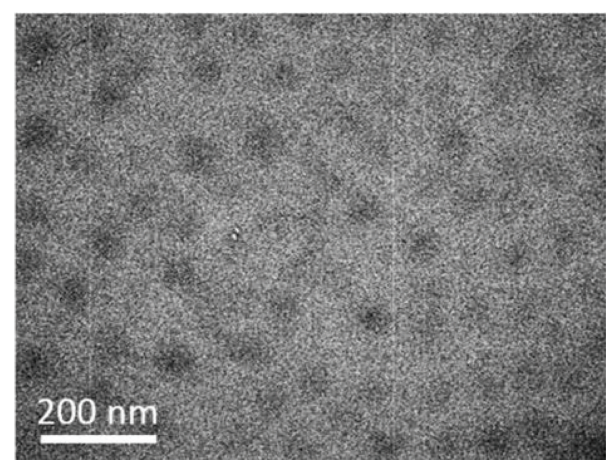

(a)

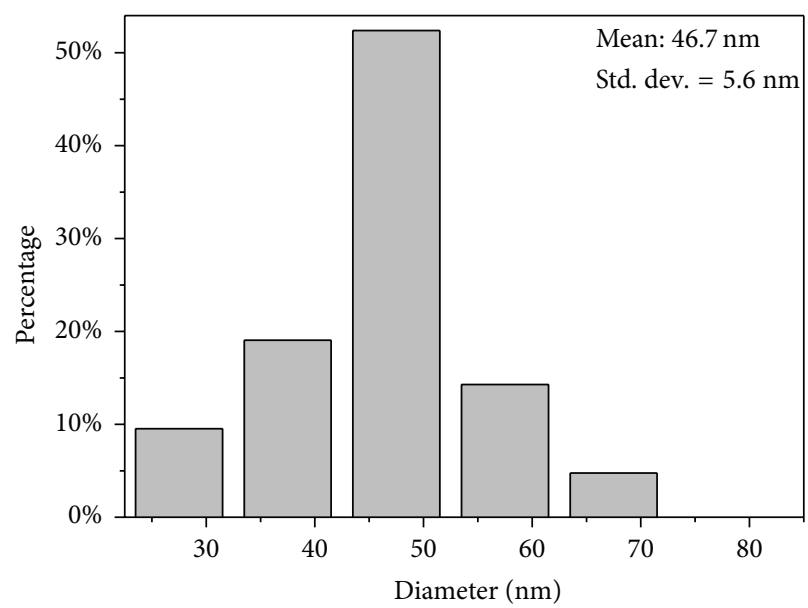

(c)

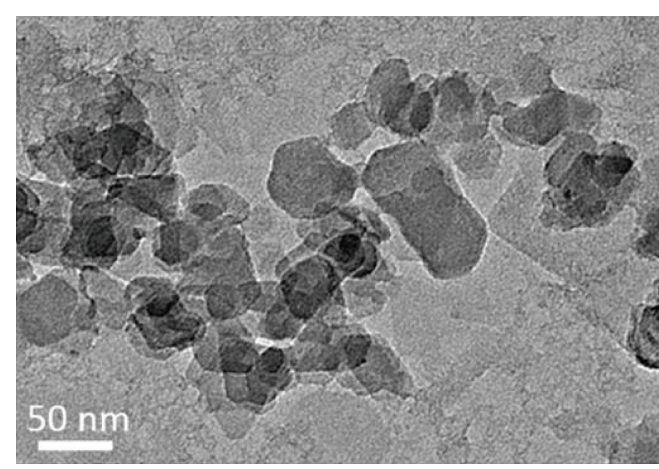

(b)

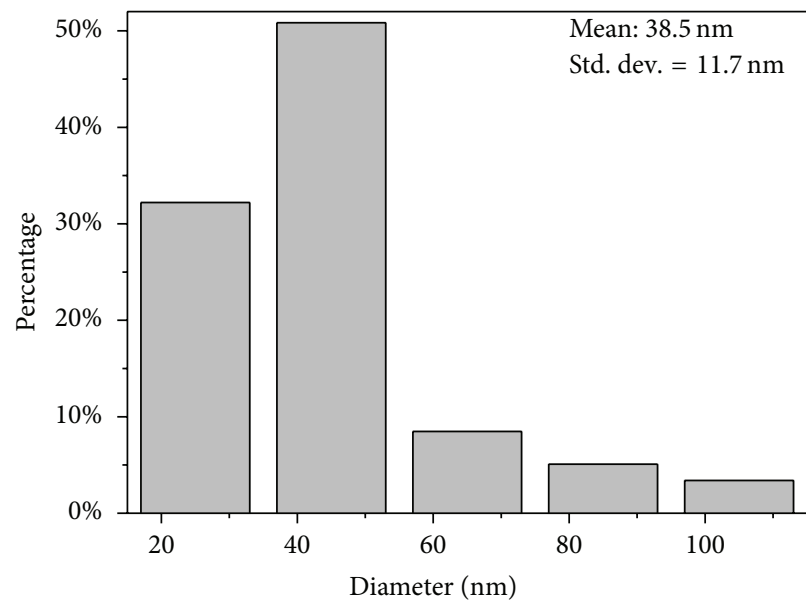

(d)

FIGURE 2: TEM images of (a) $\mathrm{C}_{18}$-GQDs and (b) p-GQDs and the size distribution histograms of (c) $\mathrm{C}_{18}$-GQDs and (d) p-GQDs.

scanning electron microscopy (FE-SEM, Quanta 600, FEI). The particle-size distribution of $\mathrm{C}_{18}$-GQDs Pickering emulsion droplets was determined by dynamic light scattering (DLS, Zetasizer Nano ZS90, Malvern Instruments).

\section{Results and Discussions}

3.1. Characterization of $C_{18}$-GQDs. Graphene quantum dots are commonly considered as mono- or few layered graphene sheets or polyaromatic species with lateral dimensions in the range of 1-100 nm [32]. TEM images of $\mathrm{C}_{18}$-GQDs were taken to verify their morphologies. In Figure 2(a), $\mathrm{C}_{18}$-GQDs showed an average size of $46.7 \pm 5.6 \mathrm{~nm}$, which resembled the results reported previously [27, 33, 34]. Comparing with p-GQDs, the low resolution/contrast of TEM image of $\mathrm{C}_{18^{-}}$ GQDs might be due to the functionalization of edge of graphene sheets, which were also observed in other polymer functionalized GQDs $[27,35]$.

Size distributions of GQDs were performed by analysis of TEM images, counting at least 100 particles. As shown in Figure 2(c), the size distribution of as-prepared $\mathrm{C}_{18}$-GQDs was relatively narrow, suggesting a uniform arrangement. The morphologies of p-GQDs were also studied by TEM, as shown in Figure 2(b). The average size of p-GQDs was $38.5 \mathrm{~nm}$ with standard deviation of $11.7 \mathrm{~nm}$, indicating that the average sizes of $\mathrm{C}_{18}$-GQDs and p-GQDs were similar. The $\mathrm{C}_{18}$-GQDs were found to be well dispersed in organic solvents such as acetone and toluene but had only limited solubility in water, which indicated successful grafting of organophilic groups on graphene nanoplatelets [35]. In contrast, $\mathrm{p}$-GQDs behaved more like graphene oxide and were well dispersed in the water due to the existence of hydrophilic carboxylic group (-COOH) [23].

The surface chemistry of $\mathrm{C}_{18}$-GQDs was further confirmed by Fourier transform infrared spectroscopy (FTIR). In Figure 3, the characteristic vibrational bands at 1090, 1370, 1710,2920 , and $3380 \mathrm{~cm}^{-1}$ were found, which can be assigned to the stretching vibrations of C-N/C-O, vibrational band of carboxylate ion $\left(-\mathrm{COO}^{-}\right)$, the overlapped vibrational absorption bands of $\mathrm{C}=\mathrm{C}$ and $\mathrm{HN}-(\mathrm{C}=\mathrm{O})-\mathrm{R}$, the $\mathrm{C}-\mathrm{H}$ stretching vibration, and the stretching vibrations of $\mathrm{C}-\mathrm{OH}$ and $\mathrm{N}-\mathrm{H}$. These results indicated that the hydrophobic groups (i.e., alkyl groups) were functionalized by amide bonds [36-38].

In order to further prove the successful synthesis of graphene quantum dots, the optical properties of $\mathrm{C}_{18}$-GQDs were examined by the ultraviolet-visible spectrophotometry (UV-Vis) and photoluminescence (PL) spectra at variable excitation wavelengths, as shown in Figure 4. The UV-Vis absorption spectrum of $\mathrm{C}_{18}$-GQDs was generally broad in the range from $350 \mathrm{~nm}$ to $600 \mathrm{~nm}$. In the inset in Figure 4, 


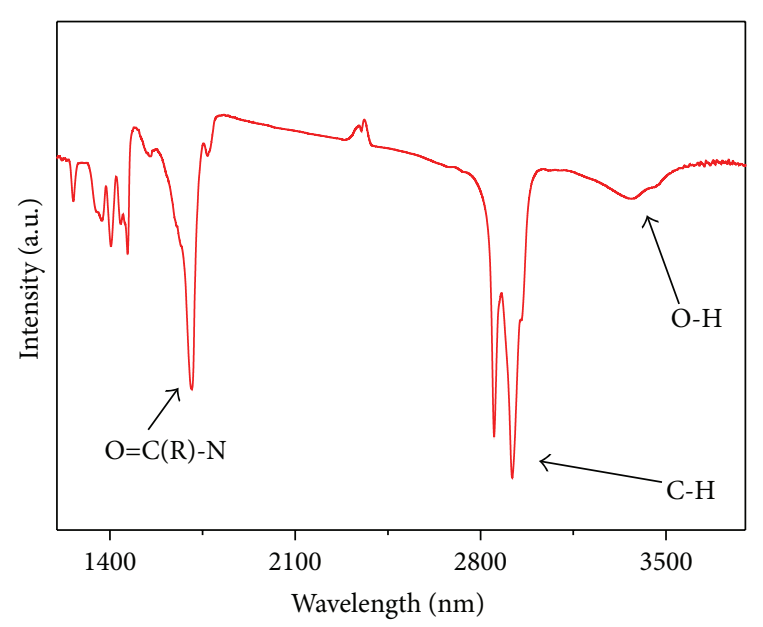

FIGURE 3: FTIR spectrum of $\mathrm{C}_{18}$-GQDs.

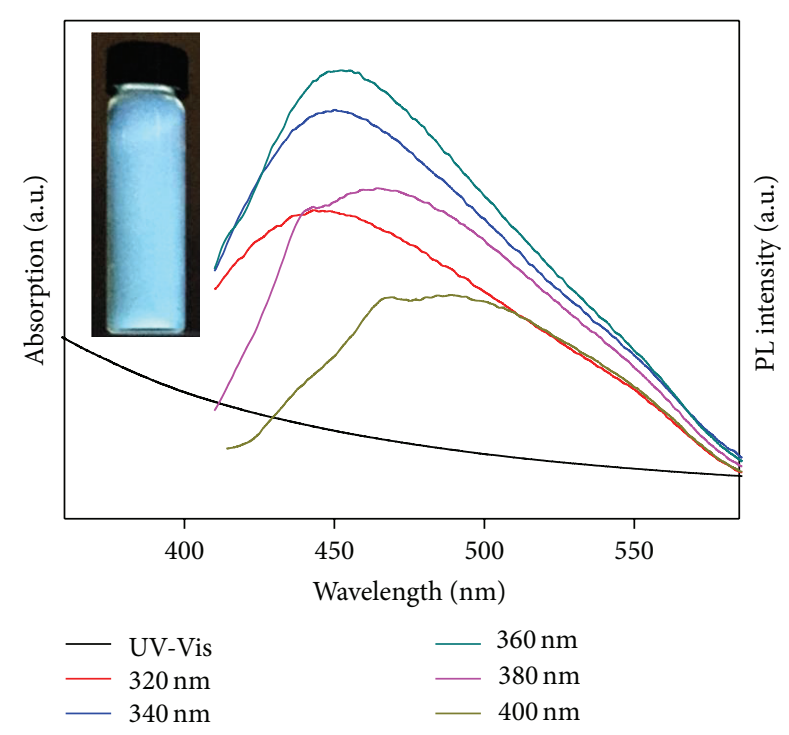

FIgURE 4: UV-Vis and PL spectra of functionalized $C_{18}$-GQDs. Inset: digital photograph of the $\mathrm{C}_{18}$-GQDs under $365 \mathrm{~nm}$ UV light.

a bright blue luminescence light was observed at a low concentration $(0.5 \mathrm{mg} / \mathrm{mL})$ under the illumination of UV $(\lambda=$ $365 \mathrm{~nm}$ ). The PL spectra of $\mathrm{C}_{18}$-GQDs were also broad and apparently dependent on the excitation wavelength. The PL peaks shifted to longer wavelengths as excitation wavelength increased from 320 to $400 \mathrm{~nm}$. In particular, $C_{18}$-GQDs showed strong blue photoluminescence and had optimal excitation and emission wavelengths at $360 \mathrm{~nm}$ and $450 \mathrm{~nm}$, respectively. Although the mechanisms underlying the PL properties of graphene quantum dots have not been completely understood yet, some theoretical modelling and calculations gave good insights into understanding PL mechanism of GQDs. For instance, by employing density-functional theory (DFT) and time-dependent DFT calculations, Sk et al. recently demonstrated that PL of a GQD could be essentially originated from the quantum confinement of conjugated $\pi$ electrons in $\mathrm{sp}^{2}$ carbon grid and can be tuned by its size, shape, edge configuration, attached chemical functionalities, and heteroatom doping and defects [39].

The wettability of $\mathrm{C}_{18}$-GQDs was determined by measuring contact angles at water-air interface. As shown in Figure 5, the contact angle for p-GQDs is $14.6^{\circ}$, indicating the high hydrophilicity due to the existence of hydrophilic carboxylic group [23]. By contrast, the contact angle of $105.8^{\circ}$ for $\mathrm{C}_{18}$-GQDs is significantly larger than $\mathrm{p}$-GQDs as well as $21.8^{\circ}$ on glass substrates, proving that functionalization of graphene quantum dots with octadecyl group introduces substantial hydrophobicity. The effective improvement of surface amphiphilicity suggested that $\mathrm{C}_{18}$-GQDs is expected to be an attractive candidate for stabilizing Pickering emulsions in comparison with highly hydrophilic p-GQDs.

3.2. Properties of the Emulsions. To examine the potential of the hydrophobic octadecyl grafted GQDs as the surfactant during emulsification, both $\mathrm{p}$-GQDs and $\mathrm{C}_{18}$-GQDs were used to prepare Pickering emulsions. At the relatively low concentration $(0.1 \mathrm{wt} \%)$, p-GQDs were observed to have rarely emulsified the dodecane-in-water droplets (Figure 6(c)), while the dodecane/water mixture in the presence of $\mathrm{C}_{18}$-GQDs formed a uniform emulsion (Figure 6(b)) that was similar to emulsions produced by amphiphilic polymer-based surfactants [31]. The poor emulsification efficiency of p-GQD indicated its overwhelming hydrophilicity, because the as-prepared p-GQDs contained a huge amount of oxygen-containing groups, including $\mathrm{C}-\mathrm{OH}$ groups and COOH groups $[23,27]$. Thus, $\mathrm{p}-\mathrm{GQD}$ s preferentially stayed in the aqueous medium. The introduction of octadecyl group on GQDs, however, induced a significant increase in the hydrophobicity and oleophilicity of the graphene quantum dots. The hydrophobic octadecyl group allowed GQDs to be strongly absorbed at the interface of water/dodecane, leading to a remarkable improvement in the emulsification efficiency. Moreover, as an effective means for estimating the emulsion stability and the emulsification effect [40], the droplet size distribution of the Pickering emulsions stabilized by $\mathrm{C}_{18}$-GQDs was studied by DLS, as shown in Figure 6(a). The average droplet size for dodecane-in-water emulsions was $263 \mathrm{~nm}$, with standard deviation of $51 \mathrm{~nm}$. Microscopic study of Pickering emulsion droplets was also performed, suggesting uniform Pickering emulsion droplets. However, well-focused microscopic images were difficult to obtain because the Pickering emulsion size falls into the range of visible light wavelength, as shown in Figure 6(d).

Figure 6(e) shows the observations of oil-in-water emulsions preferentially formed when $\mathrm{C}_{18}$-GQDs were introduced into the system at a relatively high concentration $(2.0 \mathrm{wt} \%)$ $[15,41]$. The emulsion types (oil-in-water and water-in-oil) were examined qualitatively by checking their miscibility in the water or oil phase [15]. That is, a drop of each emulsion was added to pure dodecane or to deionized water. In water, the oil-in-water emulsions dispersed but remained as drops in dodecane, and vice versa. As shown in Figure 6(e), the dodecane phase showed a dark brownish color due to the good solubility of $\mathrm{C}_{18}$-GQDs in dodecane. Besides, oil-inwater emulsions (Winsor I type) were preferred to be formed when dodecane volume fraction was higher than $20 \%$. 


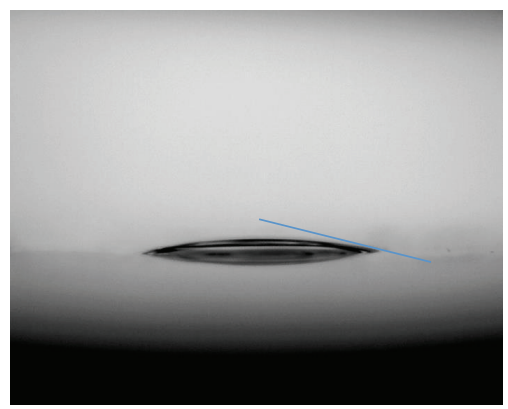

(a) $\theta=14.6^{\circ}$

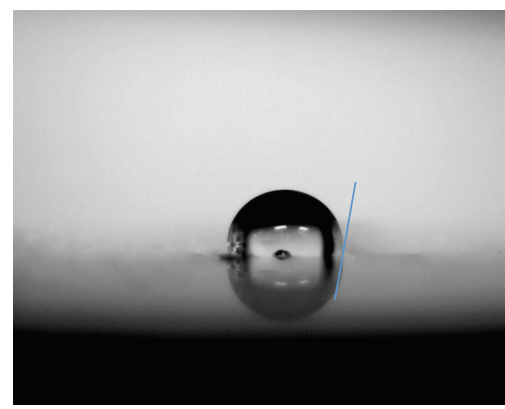

(b) $\theta=105.8^{\circ}$

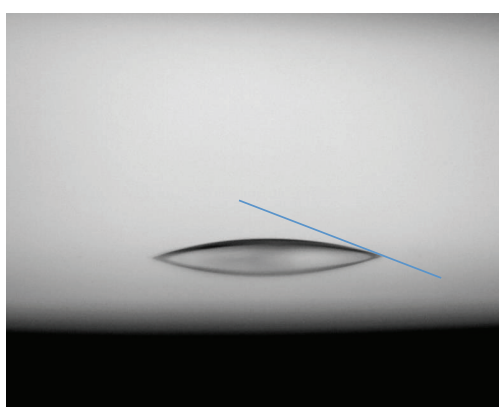

(c) $\theta=21.8^{\circ}$

Figure 5: Contact angle measurements of (a) p-GQDs, (b) $\mathrm{C}_{18}$-GQDs, and (c) bare glass substrate.

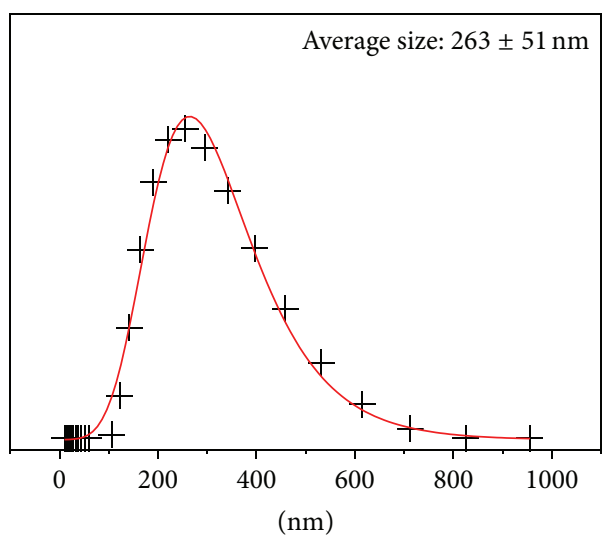

(a)

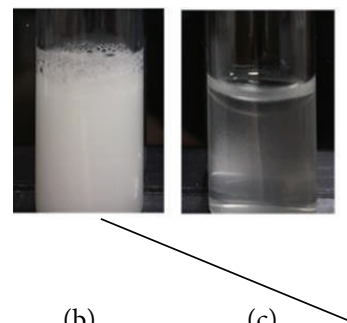

(b) (c)

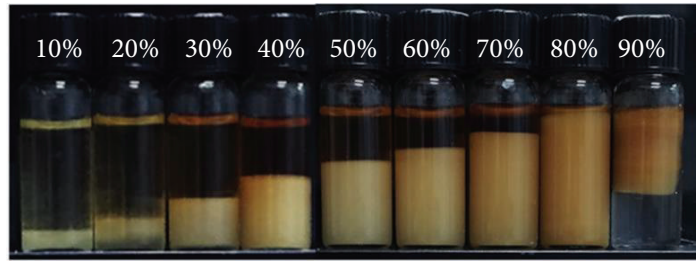

(e)

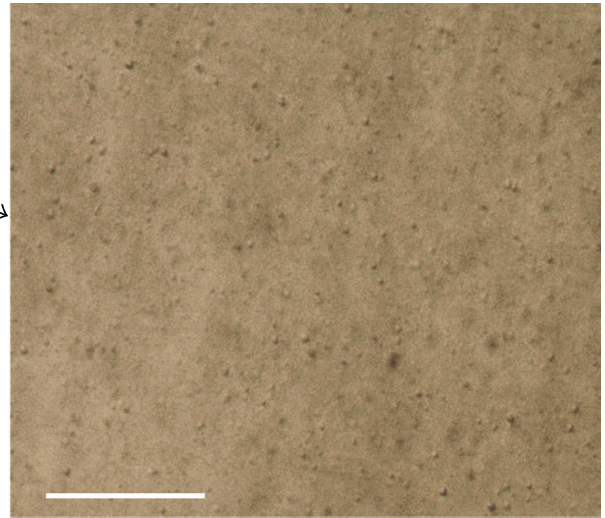

(d)

FIgURE 6: (a) The DLS spectra of Pickering emulsions stabilized by $\mathrm{C}_{18}$-GQDs. Photographic images of dodecane/water mixtures containing (b) $\mathrm{C}_{18}$-GQDs (0.1 wt\%) and (c) p-GQDs (0.1 wt\%). (d) Microscopic images of oil-in-water emulsions stabilized by $\mathrm{C}_{18}$-GQDs (0.1 wt\%) with scale bar of $50 \mu \mathrm{m}$. (e) The emulsion phase diagram of $\mathrm{C}_{18}$-GQDs (2.0 wt\%) stabilized dodecane/water mixture with various volume fraction of aqueous phase from $10 \%$ to $90 \%$ at $20^{\circ} \mathrm{C}$.

However, phase inversion occurred if oil volume fraction was low according to phase diagram $[15,41]$.

To further demonstrate the potential of $\mathrm{C}_{18}$-GQDs as a surfactant, $\mathrm{C}_{18}$-GQDs were employed to produce polymeric nanocapsules through miniemulsion polymerization method. To date, predominantly, the formation of polymeric capsules with a size of $1 \mu \mathrm{m}$ and larger is demonstrated by Pickering polymerization. However, for many practical uses, especially in medicine and high-resolution electronic inks, smaller capsules with size between 50 and $300 \mathrm{~nm}$ are of high interest [42]. To achieve successful miniemulsion polymerization, the emulsion surface is required to be stabilized by adsorption of surfactants. By using $\mathrm{C}_{18}$-GQDs as a surfactant and AIBN as an initiator, styrene was successfully emulsified and polymerized to fabricate the uniform $\mathrm{C}_{18}$-GQDs/PS microspheres, as shown in Figure 7. It was shown that the size of small microspheres was comparable to that of "thermodynamically" stable Pickering emulsions prepared by mechanical stirring of 3-methacryloxypropyltrimethoxysilane $/ \mathrm{H}_{2} \mathrm{O}$ mixture with magnetite nanoparticles [14]. An average size of the PS nanoparticles was determined to be $152 \pm 8 \mathrm{~nm}$, which was smaller than that of previously reported PS colloidal particles stabilized by graphene-based materials [11, 29, 30, 43-48]. In addition, the morphologies of $\mathrm{C}_{18}$-GQDs/PS composites are similar to that of PS nanoparticles produced by commercial surfactants [31]. These results show that $\mathrm{C}_{18^{-}}$ GQD is a promising candidate for a highly efficient surfactant forming oil-in-water Pickering emulsions. Further research is 


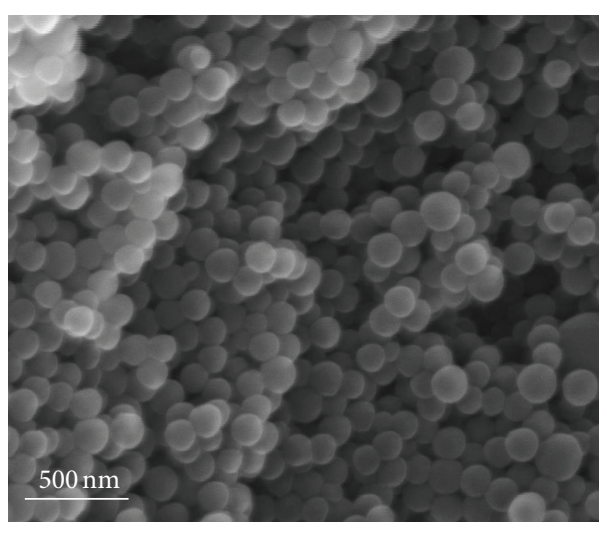

(a)

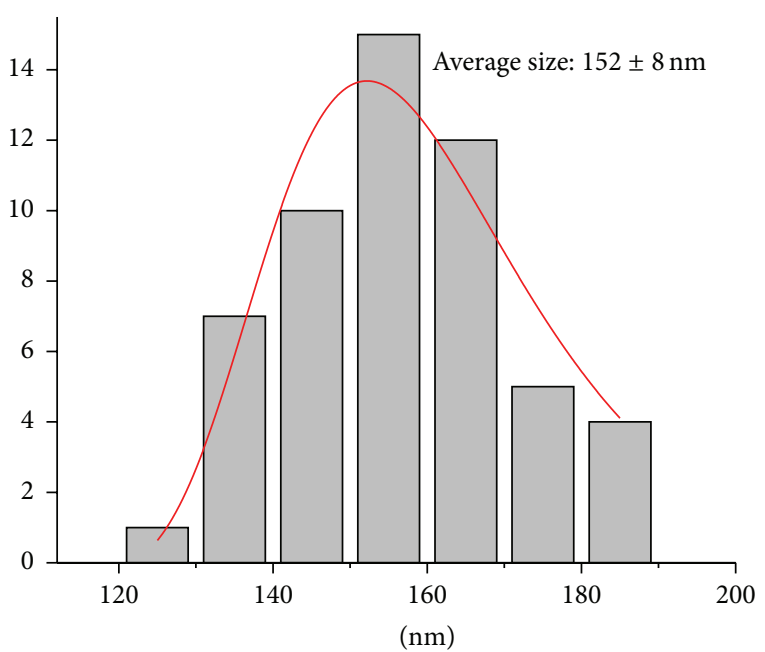

(b)

FIGURE 7: (a) SEM image of uniform PS particles stabilized by $\mathrm{C}_{18}$-GQDs and (b) the corresponding histogram of PS colloidal particles stabilized by $\mathrm{C}_{18}$-GQDs $(0.18 \mathrm{wt} \%)$.

still needed, however, to address issues such as how to utilize photoluminescent properties and how to optimize the surface chemistry of functionalized graphene quantum dots.

\section{Conclusions}

In summary, we demonstrated that octadecyl grafted graphene quantum dots ( $\mathrm{C}_{18}$-GQDs) surfactants could be used as effective emulsifiers for stabilizing Picking emulsions as well as for miniemulsion polymerization. Both the photoluminescent and excitation-dependent properties were characterized. Furthermore, by using functionalized graphene quantum dots, uniform Pickering emulsions of dodecane and water were formed with an average size of $263 \pm 51 \mathrm{~nm}$; in contrast, no stable Pickering emulsions were produced with the sole addition of nonfunctionalized p-GQDs. Phase diagram of $\mathrm{C}_{18}$-GQDs further confirmed the well-balanced amphiphilic property and suggested that water-in-oil emulsions could be created by $\mathrm{C}_{18}$-GQDs. Moreover, $\mathrm{C}_{18}$-GQDs exhibited high efficiency as surfactants to synthesize uniform $\mathrm{C}_{18}$-GQDs/PS nanocapsules via the miniemulsion polymerization. In the presence of $\mathrm{C}_{18}$-GQDs, polymeric nanocapsules with an average size of $152 \pm 8 \mathrm{~nm}$ were achieved. This facile one-pot bottom-up synthesis of amphiphilic GQDs combined with their luminescent property provides a possible direction for multifunctional carbon-based quantum dots.

\section{Competing Interests}

The authors declare that there are no competing interests regarding the publication of this paper.

\section{Acknowledgments}

This work is supported by NASA (NASA-NNX13AQ60G) and China National Petroleum \& Gas Corporation Science and Technology Development Project "Study on Asymmetric Modification Methods for Nanoplatelet Particles" (405550). The authors acknowledge the Microscope and Imaging Center (MIC) at Texas A\&M University for access to the FESEM and TEM. The FE-SEM instrument was supported by the National Science Foundation under Grant DBI-0116835. The authors also thank Dr. Mustafa Akbulut for using his dynamic light scattering instrument.

\section{References}

[1] S. U. Pickering, "CXCVI.-emulsions," Journal of the Chemical Society, Transactions, vol. 91, pp. 2001-2021, 1907.

[2] A. Stocco, E. Rio, B. P. Binks, and D. Langevin, "Aqueous foams stabilized solely by particles," Soft Matter, vol. 7, no. 4, pp. 1260 1267, 2011.

[3] U. T. Gonzenbach, A. R. Studart, E. Tervoort, and L. J. Gauckler, "Ultrastable particle-stabilized foams," Angewandte ChemieInternational Edition, vol. 45, no. 21, pp. 3526-3530, 2006.

[4] M. Rayner, D. Marku, M. Eriksson, M. Sjöö, P. Dejmek, and M. Wahlgren, "Biomass-based particles for the formulation of Pickering type emulsions in food and topical applications," Colloids and Surfaces A: Physicochemical and Engineering Aspects, vol. 458, no. 1, pp. 48-62, 2014.

[5] R. Aveyard, B. P. Binks, and J. H. Clint, "Emulsions stabilised solely by colloidal particles," Advances in Colloid and Interface Science, vol. 100-102, pp. 503-546, 2003.

[6] S. Arditty, V. Schmitt, J. Giermanska-Kahn, and F. LealCalderon, "Materials based on solid-stabilized emulsions," Journal of Colloid and Interface Science, vol. 275, no. 2, pp. 659-664, 2004.

[7] E. M. Herzig, K. A. White, A. B. Schofield, W. C. K. Poon, and P. S. Clegg, "Bicontinuous emulsions stabilized solely by colloidal particles," Nature Materials, vol. 6, no. 12, pp. 966-971, 2007.

[8] B. P. Binks, A. N. Boa, M. A. Kibble, G. Mackenzie, and A. Rocher, "Sporopollenin capsules at fluid interfaces: particlestabilised emulsions and liquid marbles," Soft Matter, vol. 7, no. 8, pp. 4017-4024, 2011. 
[9] S.-H. Kim, S. Y. Lee, and S.-M. Yang, "Janus microspheres for a highly flexible and impregnable water-repelling interface," Angewandte Chemie-International Edition, vol. 49, no. 14, pp. 2535-2538, 2010.

[10] P. Aussillous and D. Quéré, “Liquid marbles," Nature, vol. 411, no. 6840, pp. 924-927, 2001.

[11] H. Yang, D. J. Kang, K. H. Ku et al., "Highly luminescent polymer particles driven by thermally reduced graphene quantum dot surfactants," ACS Macro Letters, vol. 3, no. 10, pp. 985-990, 2014.

[12] J. H. Jiang, Y. Zhu, Z. G. Cui, and B. P. Binks, "Switchable pickering emulsions stabilized by silica nanoparticles hydrophobized in situ with a switchable surfactant," Angewandte ChemieInternational Edition, vol. 52, no. 47, pp. 12373-12376, 2013.

[13] Z. Chen, L. Zhou, W. Bing et al., "Light controlled reversible inversion of nanophosphor-stabilized pickering emulsions for biphasic enantioselective biocatalysis," Journal of the American Chemical Society, vol. 136, no. 20, pp. 7498-7504, 2014.

[14] S. Sacanna, W. K. Kegel, and A. P. Philipse, “Thermodynamically stable pickering emulsions," Physical Review Letters, vol. 98, no. 15, Article ID 158301, 2007.

[15] J. W. J. de Folter, M. W. M. van Ruijven, and K. P. Velikov, "Oilin-water Pickering emulsions stabilized by colloidal particles from the water-insoluble protein zein," Soft Matter, vol. 8, no. 25, pp. 6807-6815, 2012.

[16] M. Destribats, M. Rouvet, C. Gehin-Delval, C. Schmitt, and B. P. Binks, "Emulsions stabilised by whey protein microgel particles: towards food-grade Pickering emulsions," Soft Matter, vol. 10, no. 36, pp. 6941-6954, 2014.

[17] A. F. Mejia, A. Diaz, S. Pullela et al., "Pickering emulsions stabilized by amphiphilic nano-sheets," Soft Matter, vol. 8, no. 40, pp. 10245-10253, 2012.

[18] M. Reger, T. Sekine, T. Okamoto, K. Watanabe, and H. Hoffmann, "Pickering emulsions stabilized by novel clay-hydrophobin synergism," Soft Matter, vol. 7, no. 22, pp. 11021-11030, 2011.

[19] W. Wu, H. Chen, C. Liu, Y. Wen, Y. Yuan, and Y. Zhang, "Preparation of cyclohexanone/water Pickering emulsion together with modification of silica particles in the presence of PMHS by one pot method," Colloids and Surfaces A: Physicochemical and Engineering Aspects, vol. 448, no. 1, pp. 130-139, 2014.

[20] D. Kpogbemabou, G. Lecomte-Nana, A. Aimable, M. Bienia, V. Niknam, and C. Carrion, "Oil-in-water Pickering emulsions stabilized by phyllosilicates at high solid content," Colloids and Surfaces A: Physicochemical and Engineering Aspects, vol. 463, pp. 85-92, 2014.

[21] J. Liu, D. Yin, S. Zhang, H. Liu, and Q. Zhang, "Synthesis of polymeric core/shell microspheres with spherical viruslike surface morphology by Pickering emulsion," Colloids and Surfaces A: Physicochemical and Engineering Aspects, vol. 466, pp. 174-180, 2015.

[22] H. Li, X. He, Z. Kang et al., "Water-soluble fluorescent carbon quantum dots and photocatalyst design," Angewandte ChemieInternational Edition, vol. 49, no. 26, pp. 4430-4434, 2010.

[23] Y. Q. Dong, J. W. Shao, C. Q. Chen et al., "Blue luminescent graphene quantum dots and graphene oxide prepared by tuning the carbonization degree of citric acid," Carbon, vol. 50, no. 12, pp. 4738-4743, 2012.

[24] L. Cao, X. Wang, M. J. Meziani et al., "Carbon dots for multiphoton bioimaging," Journal of the American Chemical Society, vol. 129, no. 37, pp. 11318-11319, 2007.
[25] B. Chen, F. Li, S. Li et al., "Large scale synthesis of photoluminescent carbon nanodots and their application for bioimaging," Nanoscale, vol. 5, no. 5, pp. 1967-1971, 2013.

[26] L. A. Ponomarenko, F. Schedin, M. I. Katsnelson et al., "Chaotic dirac billiard in graphene quantum dots," Science, vol. 320, no. 5874, pp. 356-358, 2008.

[27] X. T. Zheng, A. Ananthanarayanan, K. Q. Luo, and P. Chen, "Glowing graphene quantum dots and carbon dots: properties, syntheses, and biological applications," Small, vol. 11, no. 14, pp. 1620-1636, 2015.

[28] F. Liu, S. Wang, J. Guan, T. Wei, M. Zeng, and S. Yang, "Putting a terbium-monometallic cyanide cluster into the $\mathrm{C}_{82}$ fullerene cage: TbCN@ $C_{2}(5)-C_{82}$," Inorganic Chemistry, vol. 53, no. 10, pp. 5201-5205, 2014.

[29] S. C. Thickett and P. B. Zetterlund, "Preparation of composite materials by using graphene oxide as a surfactant in $\mathrm{Ab}$ initio emulsion polymerization systems," ACS Macro Letters, vol. 2, no. 7, pp. 630-634, 2013.

[30] G. N. Yin, Z. Zheng, H. T. Wang, Q. G. Du, and H. D. Zhang, "Preparation of graphene oxide coated polystyrene microspheres by Pickering emulsion polymerization," Journal of Colloid and Interface Science, vol. 394, no. 1, pp. 192-198, 2013.

[31] K. Landfester, N. Bechthold, F. Tiarks, and M. Antonietti, "Miniemulsion polymerization with cationic and nonionic surfactants: a very efficient use of surfactants for heterophase polymerization," Macromolecules, vol. 32, no. 8, pp. 2679-2683, 1999.

[32] R. Gokhale and P. Singh, "Blue luminescent graphene quantum dots by photochemical stitching of small aromatic molecules: fluorescent nanoprobes in cellular imaging," Particle \& Particle Systems Characterization, vol. 31, no. 4, pp. 433-438, 2014.

[33] L. Li, G. Wu, G. Yang, J. Peng, J. Zhao, and J.-J. Zhu, "Focusing on luminescent graphene quantum dots: current status and future perspectives," Nanoscale, vol. 5, no. 10, pp. 4015-4039, 2013.

[34] S. J. Zhu, J. H. Zhang, S. J. Tang et al., "Surface chemistry routes to modulate the photoluminescence of graphene quantum dots: from fluorescence mechanism to up-conversion bioimaging applications," Advanced Functional Materials, vol. 22, no. 22, pp. 4732-4740, 2012.

[35] A. B. Bourlinos, A. Stassinopoulos, D. Anglos, R. Zboril, M. Karakassides, and E. P. Giannelis, "Surface functionalized carbogenic quantum dots," Small, vol. 4, no. 4, pp. 455-458, 2008.

[36] J. Wang, C.-F. Wang, and S. Chen, "Amphiphilic egg-derived carbon dots: rapid plasma fabrication, pyrolysis process, and multicolor printing patterns," Angewandte Chemie-International Edition, vol. 51, no. 37, pp. 9297-9301, 2012.

[37] D. M. Hodgson, S. Man, K. J. Powell et al., "Intramolecular oxonium ylide formation-[2,3] sigmatropic rearrangement of diazocarbonyl-substituted cyclic unsaturated acetals: a formal synthesis of hyperolactone C," Journal of Organic Chemistry, vol. 79, no. 20, pp. 9728-9734, 2014.

[38] W. T. Wu, Z. H. Zhang, and L. S. Liebeskind, "In situ carboxyl activation using a silatropic switch: a new approach to amide and peptide constructions," Journal of the American Chemical Society, vol. 133, no. 36, pp. 14256-14259, 2011.

[39] M. A. Sk, A. Ananthanarayanan, L. Huang, K. H. Lim, and P. Chen, "Revealing the tunable photoluminescence properties of graphene quantum dots," Journal of Materials Chemistry C, vol. 2, no. 34, pp. 6954-6960, 2014.

[40] W. R. Liu, D. J. Sun, C. F. Li, Q. Liu, and J. Xu, "Formation and stability of paraffin oil-in-water nano-emulsions prepared by 
the emulsion inversion point method," Journal of Colloid and Interface Science, vol. 303, no. 2, pp. 557-563, 2006.

[41] B. P. Binks and S. O. Lumsdon, "Catastrophic phase inversion of water-in-oil emulsions stabilized by hydrophobic silica," Langmuir, vol. 16, no. 6, pp. 2539-2547, 2000.

[42] F. Tiarks, K. Landfester, and M. Antonietti, "Preparation of polymeric nanocapsules by miniemulsion polymerization," Langmuir, vol. 17, no. 3, pp. 908-918, 2001.

[43] K.-Y. A. Lin, H. T. Yang, C. Petit, and W.-D. Lee, "Magnetically controllable Pickering emulsion prepared by a reduced graphene oxide-iron oxide composite," Journal of Colloid and Interface Science, vol. 438, pp. 296-305, 2015.

[44] S. D. Kim, W. L. Zhang, and H. J. Choi, "Pickering emulsionfabricated polystyrene-graphene oxide microspheres and their electrorheology," Journal of Materials Chemistry C, vol. 2, no. 36, pp. 7541-7546, 2014.

[45] S. H. C. Man, N. Y. M. Yusof, M. R. Whittaker, S. C. Thickett, and P. B. Zetterlund, "Influence of monomer type on miniemulsion polymerization systems stabilized by graphene oxide as sole surfactant," Journal of Polymer Science Part A: Polymer Chemistry, vol. 51, no. 23, pp. 5153-5162, 2013.

[46] J. Sun and H. Bi, "Pickering emulsion fabrication and enhanced supercapacity of graphene oxide-covered polyaniline nanoparticles," Materials Letters, vol. 81, pp. 48-51, 2012.

[47] M. Y. Tang, T. Wu, X. Y. Xu, L. Zhang, and F. Wu, "Factors that affect the stability, type and morphology of Pickering emulsion stabilized by silver nanoparticles/graphene oxide nanocomposites," Materials Research Bulletin, vol. 60, pp. 118-129, 2014.

[48] W. Y. Zhai, G. P. Li, P. Yu, L. F. Yang, and L. Q. Mao, "Silver phosphate/carbon nanotube-stabilized pickering emulsion for highly efficient photocatalysis," Journal of Physical Chemistry C, vol. 117, no. 29, pp. 15183-15191, 2013. 

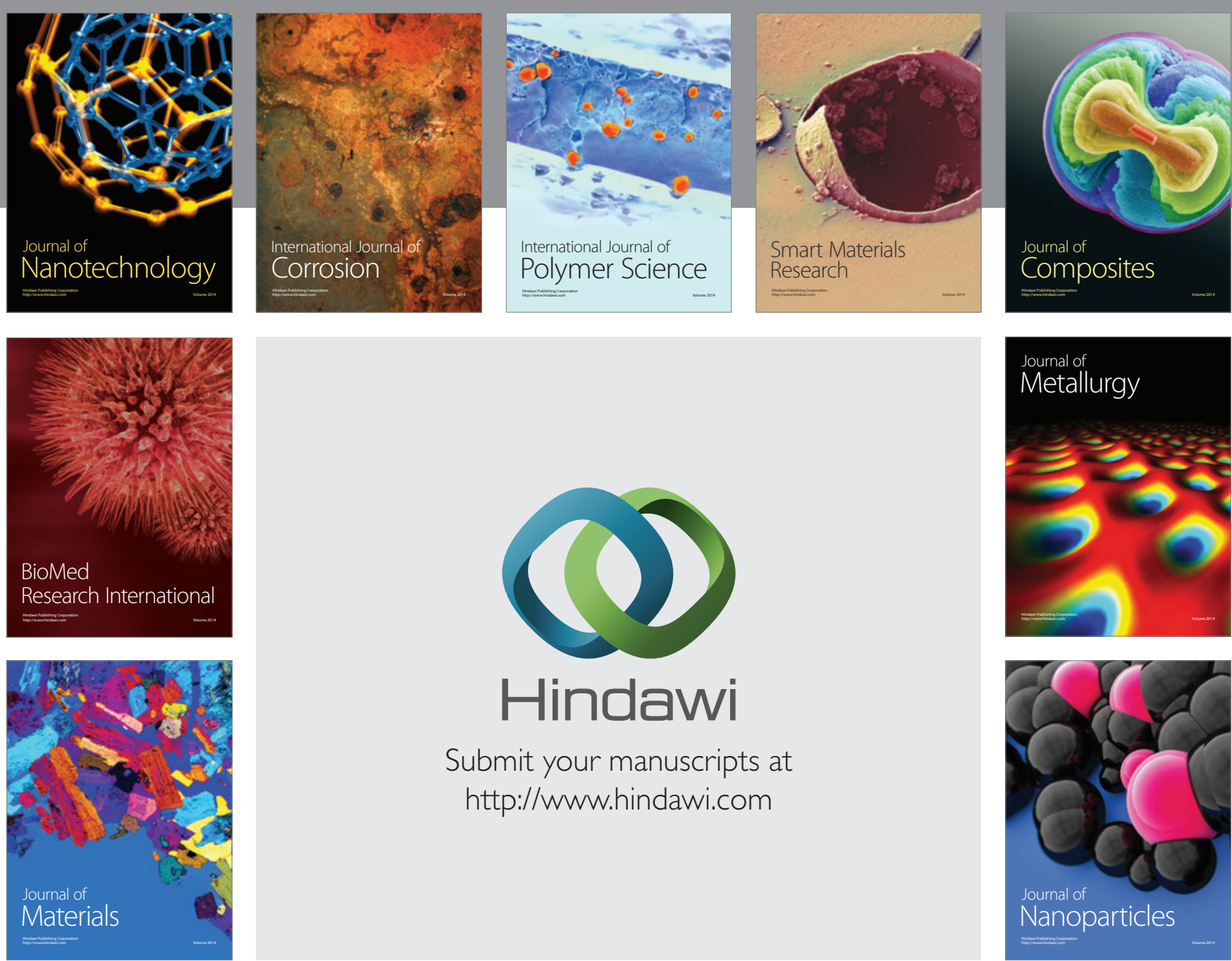

\section{Hindawi}

Submit your manuscripts at

http://www.hindawi.com

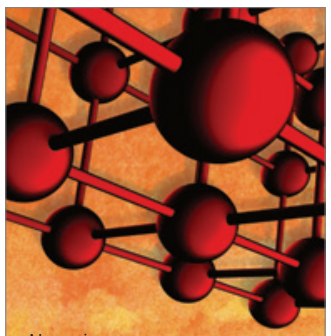

Materials Science and Engineering
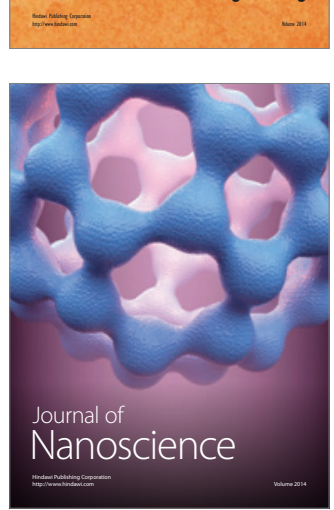
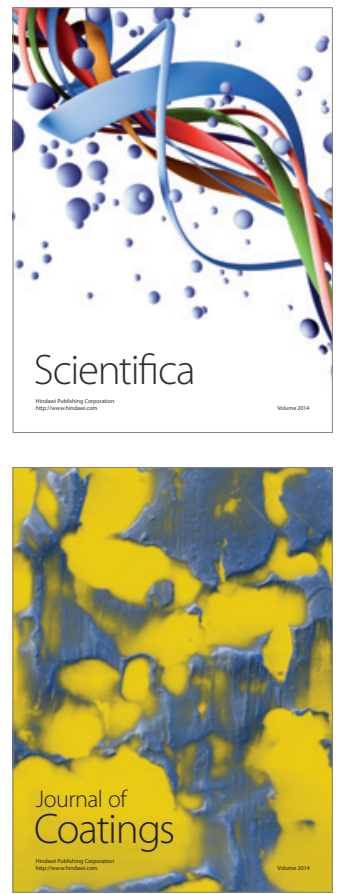
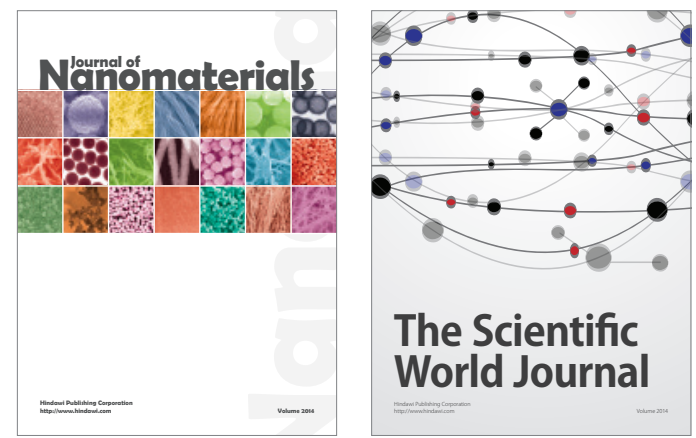

The Scientific World Journal
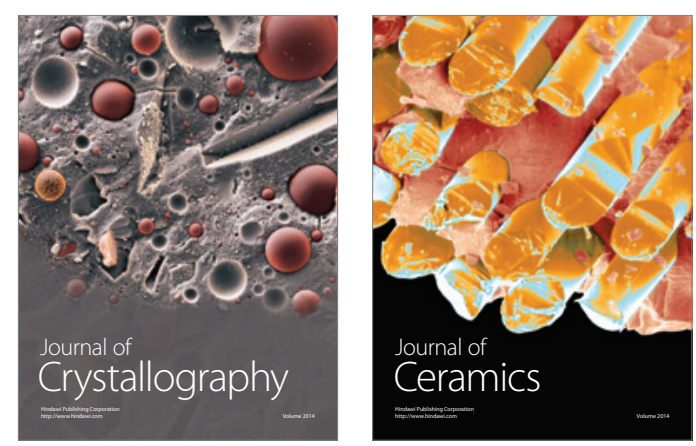
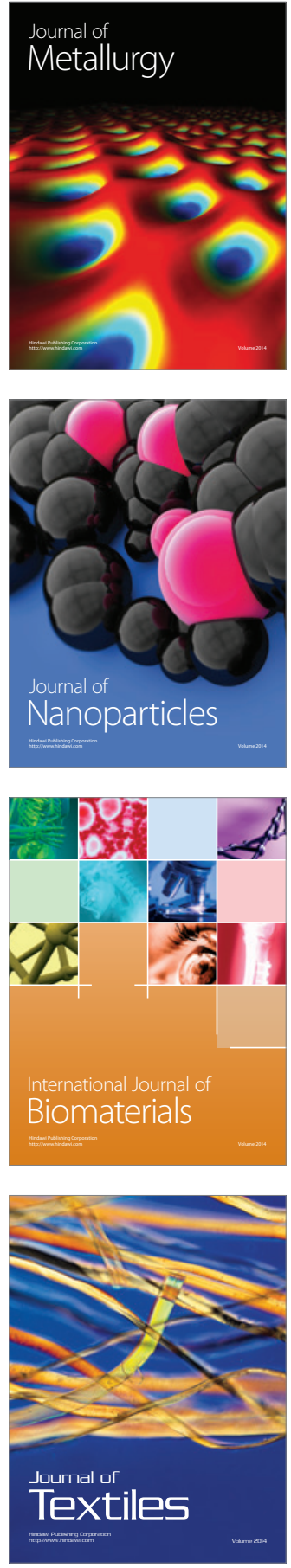